\title{
Effect Comparative Analysis of Crop Protection Electrostatic Spray and Pressure Spray
}

\author{
He Yujing ${ }^{1 a}$, Li Xiangfu ${ }^{2 b}$,Sun Weiping ${ }^{3 c}$,Li Baoqian ${ }^{4 d}$ \\ ${ }^{1,2,4}$ Henan Agricultural University, Zhengzhou, China \\ ${ }^{3}$ Henan Zhongtian Hi-Tech Intelligent Technology Development Co.,Ltd, Zhengzhou, China

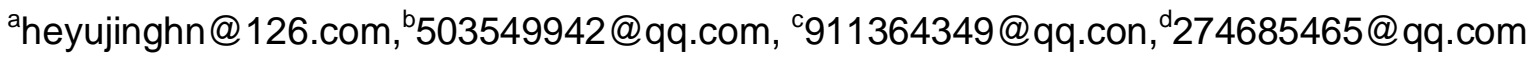

Keywords: Electrostatic spray; Droplets Diameter; Deposition Performance; Coverage Rate.

Abstract. We can get conclusion from experimental effect comparison of electrostatic spray and general pressure spray that electrostatic spray can reduce droplet diameter and narrow droplet spectrum scope as well as increase droplet evenness; influenced by electrostatic absorptive effect, droplet coverage rate on the target crop front leaf have been increased, with especially obvious increase of coverage rate on the target crop back leaf.

\section{Introduction}

Presently, chemical plant protection spray is one of important methods in pest control agriculturally, which is generally to pressurize pesticide liquid and crush into fine droplets with liquefying applicator and then spray the droplets on the plant leaves, being influenced by pressure and environmental wind power, evenness, absorption, deposition rate and on-target effect of the droplets cannot achieve ideal effect. Electrostatic spray technique is to apply HV electrostatic so as to make one electrostatic field between applicator and spray target, while pesticide liquid after atomization of applicator is charged in different charging methods and then shapes group charged droplets (droplet cloud), then the droplets are absorbed on the parts of target with orienteering movement under compound impacts of electrostatic field and other external forces, and accordingly take effects of high deposition efficiency and low droplet drift and loss, especially evenness and on-target effect of the droplets can be improved obviously. The author of this paper has taken experiments of spraying effect of two sprayers and then got better understanding for electrostatic spray features by comparison, which has offered technical theoretical basis for promotion of electrostatic spray, and promoted the application of the scientific results in agricultural production greatly, as well as offered references for improvement of future electrostatic spraying installation, perfect of traditional sprayer structure, and improvement of performance.

\section{Experimental Material \& Method}

Experimental equipments are one common domestic 3WBS-16A sprayer, one 3JWB electrostatic sprayer; XSP-33 type (100X-1600X) monocular microscope; Canon 60D kit set (18-135mm) digital camera; and CN61M/BT-9300H type laser particle sizer. In order to get easy observation of experimental results, we have adopted simulated medicine liquid which has added red ink to improve resolution capacity.

We adopt three types of spray targets:

( 1 ) Flour-strip. It is mainly to test droplet size and evenness and get precise target reception time while experiment, especially for instantaneous fulfillment which can not make droplet accumulation, we must take instantaneous picture on reception and observe it under microscope, and finish counting and measuring and calculating of droplet size.

( 2 )Use empty computer data disk. Make use of electromagnetic induction features to mainly test droplet absorptive capacity and deposition rate in the same method as above. 
( 3 )Plant leaves with large actual size in the field. It is mainly to test droplet on-target effect, front and back receiving quantity, deposition rate and droplet drifting condition. Data test method is the same as $(1)$.

\section{Analysis for Experimental Results}

\section{Comparative Test and Effect Analysis for Size}

The following Fig. 1 is samples collected from two sprayers under experimental condition.
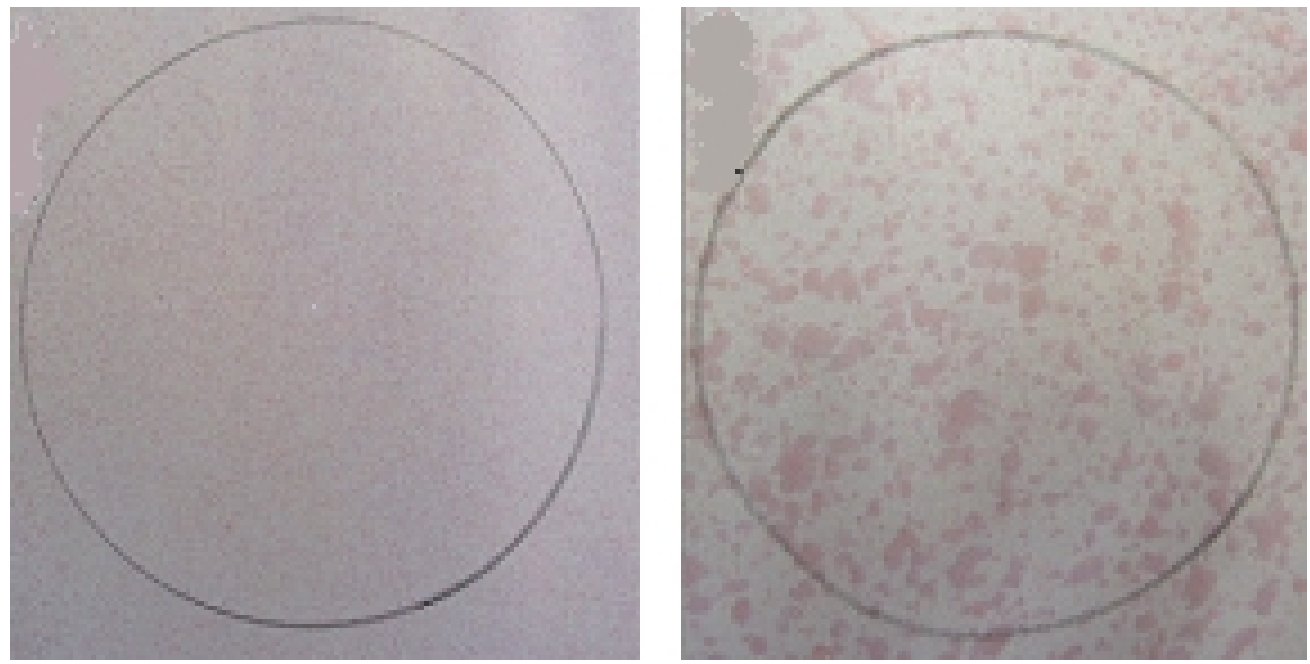

a. Electrostatic Spraying

b. Common Spraying

Fig. 1 Samples Collected from Two Sprayers

We have used laser particle sizer to measure droplet size from 3JWB-16A electrostatic sprayer and 3WBS-16A sprayer respectively, and then drawn Fig. 2 and Fig. 3 size distribution graph according to measured data:

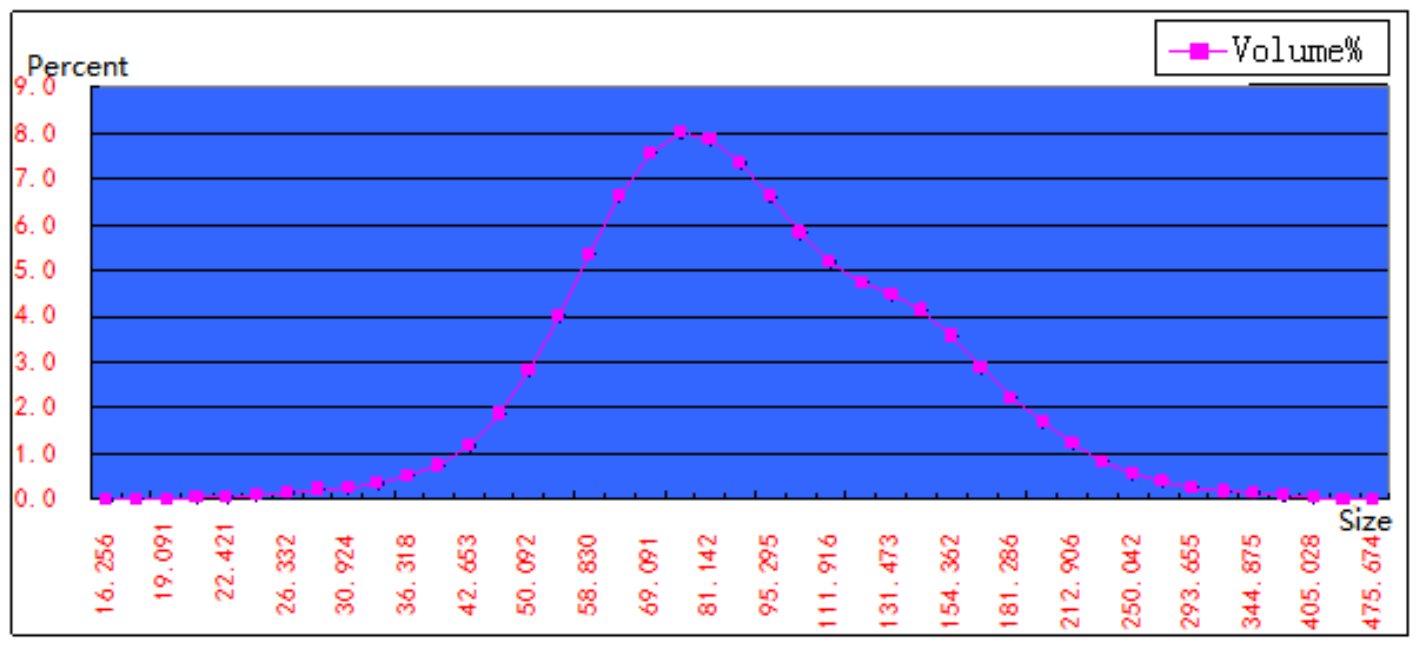

Fig.2 Droplet Size Distribution Graph of Electrostatic Sprayer 


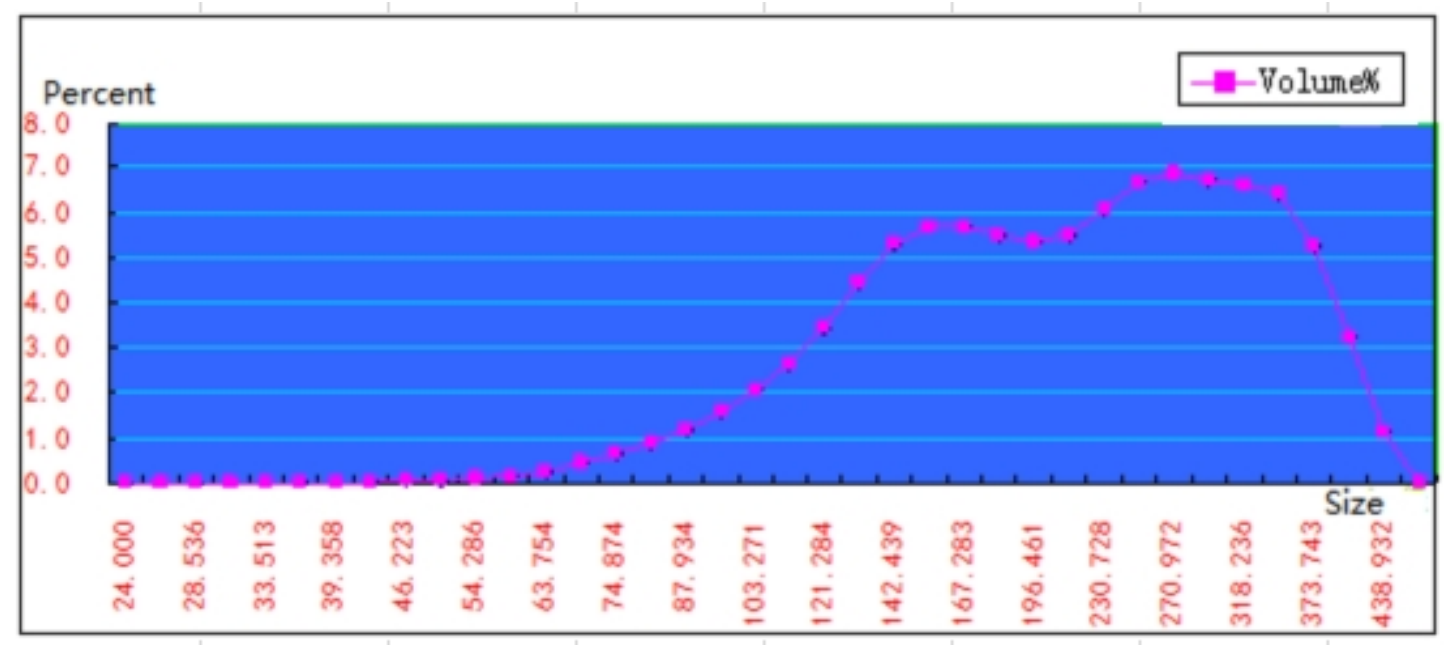

Fig.3 Droplet Size Distribution Graph of Common Sprayer

We get the following data based on analysis for free distribution:

Charged Droplet: D10 $=52.021 \mu \mathrm{m}$; D50 $=83.184 \mu \mathrm{m}$; D90 $=156.293 \mu \mathrm{m}$.

Common Droplet: P10 $=111.937 \mu \mathrm{m} ; \mathrm{P} 50=210.166 \mu \mathrm{m} ; \mathrm{P} 90=343.902 \mu \mathrm{m}$.

So volume medium diameter (VMD) of charged droplet is D50=83.184 $\mu \mathrm{m}$, and volume medium diameter (VMD) of common droplet is $\mathrm{P} 50=210.166 \mu \mathrm{m}$, namely charged droplet size is reduced by 2.5 times based of no-charged droplet, with the size is among the very droplet size scope with the best control size. If the head and end is ignored for minimum proportion in size spectra, then their size spectra are: $\mathrm{D} 90-\mathrm{D} 10=104.272 \mu \mathrm{m} ; \mathrm{P} 90-\mathrm{P} 10=231.965 \mu \mathrm{m}$ respectively., namely size spectra of charged droplet is reduced by over two times of that of common droplet, droplet evenness is certainly improved accordingly.

\section{Analysis for Droplet Coverage Density Effect}

Droplet quantity deposited in unit area is called droplet coverage density, which is generally represented by droplet quantity on $1 \mathrm{~cm} 2$ crop surface. Droplet coverage density can be measured with Caromicert test paper.

Droplet density distribution evenness is obtained from calculation of distribution mutation rate. Smaller the highest and lowest droplet density distribution mutation rate value means evener droplet density distribution and better droplet quality. If both values are zero, then it indicates droplet density distribution is the same everywhere.

Highest Droplet Density Mutation Rate $=\underline{\text { Highest Droplet Density - Average Droplet Density }}$ Average Droplet Density

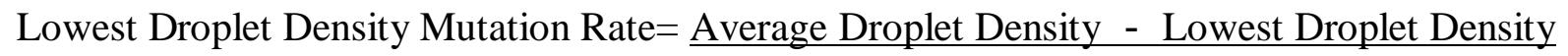

Average Droplet Density

Electrostatic spray distribution mutation rate is obtained from experimental calculation, as in Eq. (1),(2).

Highest Droplet Density Mutation Rate $=\frac{220-206}{206} \times 100 \%=6.8 \%$

Lowest Droplet Density Mutation Rate $=\frac{206-193}{206} \times 100 \%=6.3 \%$

From comparison and analysis for the above two groups of experiments and according to droplet coverage density definition and calculation formula of highest and lowest droplet density distribution mutation rate and the symbolic meanings, we can get that: coverage density of charged droplet (206 
drop/cm2) is larger than coverage density of no-charged droplet (35 drop/cm2), with droplet density distribution evenness increased significantly.

\section{Absorptive Power between Droplet and Target Object}

Use empty computer data disk to test absorptive power between droplet and target object,as shown in Figure 4-1 and Figure 4-2. We can see that two parts on the disk are in sharp contrast, and charged droplet can be absorbed on the clean disk and hard to shake off.

Two mainly reasons for good absorptive performance of charged droplet for target object are: firstly, absorptive power of small size droplet for the disk, secondly, influence of electrostatic attraction. Absorptive power of droplet with static electricity is strengthened greatly; Droplet with static electricity can get reduced droplet size, evener distribution on the disk, and more significant increased droplet coverage density.

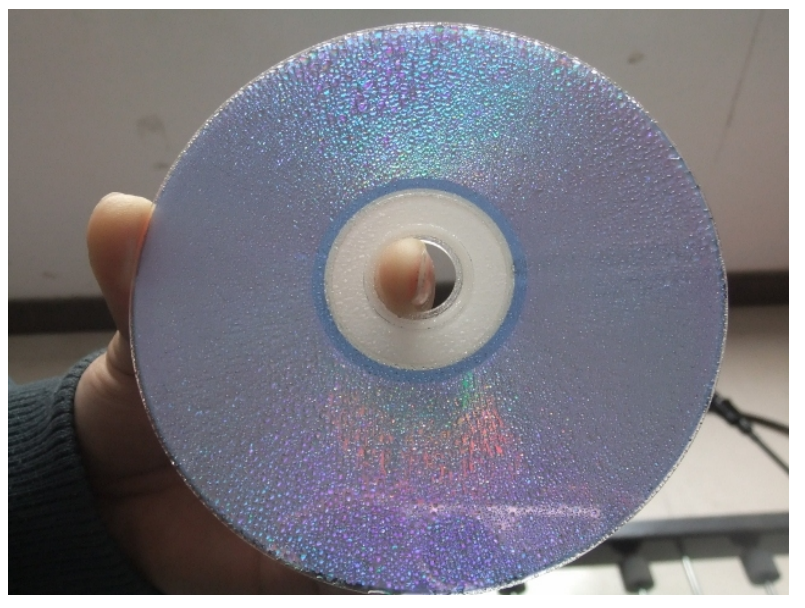

Fig. 4-1 Absorptive Effect of Droplet with Static Electricity

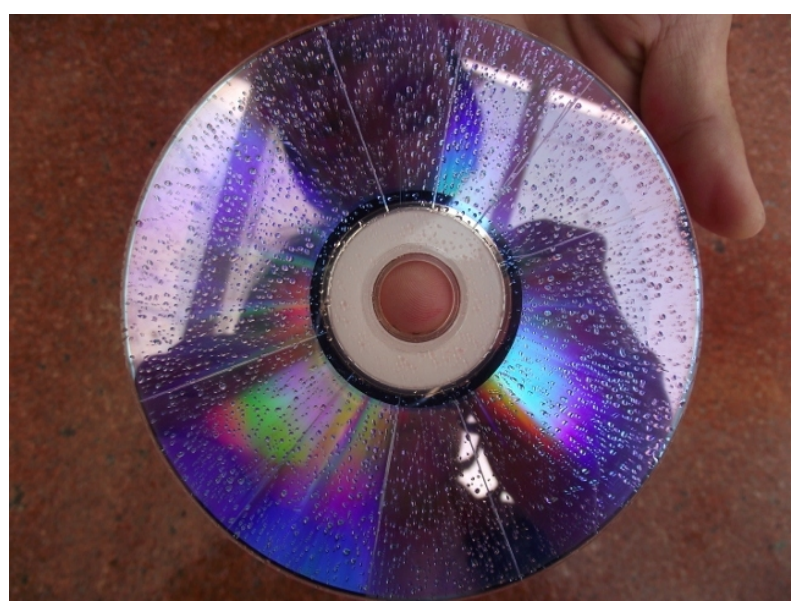

Fig. 4-2 Absorptive Effect of Droplet without Static Electricity

\section{Experimental Results Analysis for Front and Back Deposition Rate and Coverage Rate of Target Object}

In order to test orienteering drift of droplet resulted from electrostatic spray and target object absorption and deposition effect, we collect experimental images from field crop leaf surface, and take instantaneous experiment under normal state and immediately take photos of plant leaf droplet with camera. Local magnified photos refer to Fig. 5, which are sampling conditions of front and back leaf under electrostatic spray respectively.
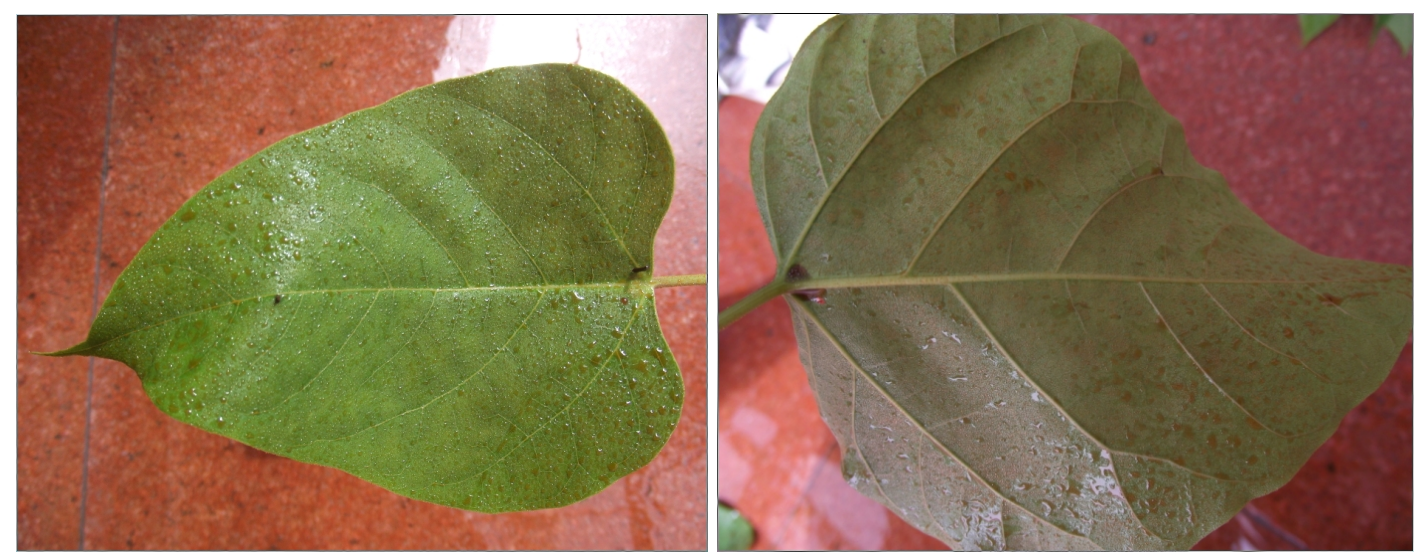

Fig. 5 Face (Left) and Back Effect Image of Leaf under 
After photograph, immediately observe droplet quantity on sampled leaf of each sampled leaf with microscope (sampling area for test is $15 \mathrm{~cm} 2$ ). Record droplet quantity in experimental result in Table 1 by sampling group.

Table 1 Droplet Coverage Rate under Common Spray and Electrostatic Spray

\begin{tabular}{cccc}
\hline Spray Method & Unit & Front & Back \\
\hline Spray & Droplet Quantity (Drop) & & \\
Electrostatic & Droplet Quantity (Drop) & 795 & 0 \\
Spray & Coverage Rate (Drop & 1080 & 165 \\
Common Spray & $/ \mathrm{cm}^{2}$ ) & 53 & 0 \\
Electrostatic & Coverage Rate (Drop & 72 & 11 \\
Spray & $/ \mathrm{cm}^{2}$ ) & & \\
\hline
\end{tabular}

Known from Table 1, droplet quantity on the front face of copy paper is 795 drops and 0 drop on the back face of copy paper under common spray. Droplet quantity on the front face of copy paper is 1,080 drops and 165 drops on the back face of copy paper under electrostatic spray. Electrostatic spray has more droplet quantity on the front and back face than common spray. Meanwhile by contrast of increasing condition of front and back coverage rate, electrostatic spray is increased significantly than common spray in terms of front coverage rate, i.e. from $53 \mathrm{drop} / \mathrm{cm} 2$ to 72 drop/cm 2 by $26.4 \%$, while back coverage rate is increased from 0 to $11 \mathrm{drop} / \mathrm{cm} 2$, which gets great difference from 72 drop/cm2 front coverage rate of electrostatic spray and $84.7 \%$ smaller than front coverage rate. The reason is that droplet needs certain drifting period to absorb and deposit on the back face. Droplets have started deposition on the back face of the leaf under instantaneous experiment of electrostatic spray.

\section{Conclusion}

We get the following conclusion from experiment and data statistical analysis for traditional sprayer and electrostatic sprayer:

(1) Polar theory of electrostatic spray for charged droplet has been testified. Droplets under the influence of electrostatic power make orienting movement and then are absorbed on the target crop. Droplets are influenced comprehensively by electrostatic power, gravity, air buoyancy and inclined force in the process of moving to target crop, but main influencing power is electrostatic power and gravity for droplet deposition. While for small droplets, droplet gravity is far smaller than electrostatic, so electrostatic power can control droplet movement and make droplet move to the target.

(2) We can get conclusion from contrastive experiments of droplet diameter under electrostatic spray and common spray: electrostatic spray can reduce droplet diameter and droplet spectra and increase droplet evenness.

(3) Main performances of electrostatic spray include small atomized droplet diameter and high evenness, which mainly for droplet surface charge under electrostatic impact leads to reduced droplet surface tension and accordingly reduced atomized drag. At the same time, repulsive force and electrostatic power among charge droplets have changed droplet surface pressure difference, which is good for fining of droplets; secondly, droplets get better deposition features, which is mainly represented by high droplet coverage rate.

(4) Electrostatic spray can increase coverage rate of leaf drop, especially front face of target crop leaf gets significant increase of coverage rate, stronger penetrating power of electrostatic spray droplet, high on-target rate, less small droplet loss and evener coverage, so it can greatly improve spray effect, reduce pesticides application amount and environmental pollution of pesticides, as well as improve application safety of pesticides. 


\section{References}

[1] Wang Tao, He Jinge, Liao Yulan, Et Al. Development Research of Plant Protection Spray Machinery [J]. Auhui Agricultural Science Bulletin, 2008, 14(21):163-164.

[2] Kakuyon Asano, electrostatic charge distribution [J]. Disease Prevention for Plant (Japan), 1986, 40(3): $12-15$.

[3] Hou Jinrong, Wang Zeyong. How to spray pesticides to save pesticides and strengthen effects [J]. New Agricultural Technology, 2005(6):10-11.

[4] Yang Ying, Ge Xiaokang. plant protection machinery and its application techniques current condition and measures of Xinjiang Corps [J]. Journal of Agricultural Mechanization Research, 2005(5).

[5] Dai Feifei. Development Trend of Pesticide Application Technology in China [C]// 40th Anniversary Celebration for China Association of Agricultural Machinery Manufacturers \& Proceedings of Annual Conference in 2003. Beijing: China Association of Agricultural Machinery Manufacturers, 2003.

[6] Zheng Lihan, Wu Chundu. Application of Electrostatic Technology in Agriculture [J]. Journal of Agricultural Mechanization Research, 2004(6):204-205.

[7] Zheng Jiaqiang, Xu Youlin. Electrostatic Pesticide Spraying Technology [J]. Electrostatics, 1994, 9 (2): 8-11.

[8] Yu Yongchang, Wang Baohua. Review on Electrostatic Spraying Technique [J]. Agriculture \& Technology, 2004, 24(4):190-195.

[9] Yu Yang. Research on Charging Effect of ULV Electrostatic Spraying Machine [J]. Yunan Agricultural University 1995.9 X 3rd Period 202 206.

[10] Qin Haoquan, Et Al. Research on Electrostatic Spray Compound Dust Removal Technology [C]. 8th Proceedings of Annual Electrostatic Conference for Chinese Physics Society (1999.8) 61 64.

[11] Wang Ze. Charged Gas-Solid Two-Phase Flow and Application in Plant Protection Engineering: [Doctor's Degree Dissertations]. Jiangsu University of Science and Technology, 1994.

[12] Mechanical Engineering Research Institute. Equipment for Crop Protection-General Test Methods [M]. P.R.C. Machine Industry Standard. 2000.4. 
